\title{
Predicción y simulación, mediante lógica difusa, de la temperatura de salida del arrabio en un horno alto(*)
}

\author{
Miguel Angel Romero*, Juan Jiménez*, Javier Mochón*, José Luis Menéndez**, \\ Antonio Formoso* y Francisca Bueno*
}

\begin{abstract}
Resumen El presente arículo describe el desarrollo y posterior validación de un modelo para predecir la temperatura del arrabio de un horno alto, basado en lógica difusa. El modelo emplea, como variables de entrada, las variables de control propias del horno: caudal de viento, humedad, inyección de carbón, adición de oxígeno, etc. y obtiene, como resultado, el valor de la temperatura del arrabio producido por el horno, con un horizonte de predicción de 40 min. Las variables empleadas para el desarrollo del modelo se obtuvieron de los datos suministrados por lo sensores de un horno alto real. Fue preciso analizar y tratar adecuadamente dichos datos antes de introducirlos en el modelo. Se prestó especial atención a la correlación temporal de los datos adecuando mediante interpolación los distintos períodos de muestreo. El modelo se entrenó utilizando dos tipos de algoritmos: un sistema de inferencia neuro-difuso adaptativo (ANFIS) y el Agrupamiento Sustractivo.
\end{abstract}

Palabras clave: Horno alto. Lógica difusa. Predicción. Simulación. Temperatura del arrabio.

\section{Hot metal temperature prediction and simulation by fuzzy logic in a blast furnace}

\begin{abstract}
This work describes the development and further validation of a model devoted to blast furnace hot metal temperature forecast, based on Fuzzy logic principles. The model employs as input variables, the control variables of an actual blast furnace: Blast volume, moisture, coal injection, oxygen addition, etc. and it yields as a result the hot metal temperature with a forecast horizon of forty minutes. As far as the variables used to develop the model have been obtained from data supplied by an actual blast furnace sensors, it is necessary to properly analyse and handle such data. Especial attention was paid to data temporal correlation, fitting by interpolation the different sampling rates. In the training stage of the model the ANFIS (Adaptive Neuro-Fuzzy Inference System) and the Subtractive Clustering algorithms have been used.
\end{abstract}

Keywords Blast furnace. Fuzzy logic. Forecasting. Simulation. Hot metal temperature.

\section{INTRODUCCIÓN}

El control de la temperatura del arrabio es tan complejo que, en la actualidad, la mayoría de los que trabajan en los hornos altos, según sus experiencias y criterios, son los que realizan las actuaciones que se necesitan para intentar mantener la temperatura lo más próxima a un valor de consigna determinado con un cierto margen de maniobra. Debido a esto, la temperatura del arrabio sufre unas oscilaciones muy grandes pudiendo llegar a tener desviaciones de hasta $40^{\circ} \mathrm{C}$ por defecto y por exceso sobre el valor de consigna.

Las aproximaciones convencionales de la ingeniería, que sólo pueden utilizar información numérica, son incapaces de aprovechar la experiencia de los trabajadores del horno alto, ya que la forma que tienen de expresar su conocimiento es por medio de condicionales. Por ejemplo, si la temperatura del arrabio sube o baja, entonces hay que aumentar o disminuir la humedad, respectivamente. El conjunto de estas oraciones condicionales se

(•) Trabajo recibido el día 20 de mayo de 1999 y aceptado en su forma final el 24 de noviembre de 1999.

$(*)$ Centro Nacional de Investigaciones Metalúrgicas, CENIM (CSIC). Avda. Gregorio del Amo, 8. 28040 Madrid (España).

(**) ACERALIA. Aptdo. 570.33280 Gijón. Asturias (España). 
denomina información lingüística. Los sistemas basados en lógica difusa son muy importantes porque permiten la incorporación, tanto de la información numérica como de la información lingüística, a la ingeniería de una forma sistemática.

Dentro de los sistemas difusos, en este trabajo se ha empleado un sistema adaptativo que incorpora un algoritmo de entrenamiento donde el sistema se construye a partir de un conjunto de reglas SIENTONCES difusas, y el algoritmo de entrenamiento ajusta los parámetros y las estructuras del sistema de lógica difusa basándose en información numérica. Por tanto, en los sistemas adaptativos, las reglas se generan automáticamente mediante un entrenamiento.

En la actualidad, la lógica difusa se está empleando en muchas aplicaciones de ingeniería ${ }^{[1]}$ : control de un avión (Rockwell Corp.), control de dirección (Nissan), transmisión automática (Nissan, Subaru), lanzadera espacial (NASA), ajuste de la imagen de TV (Sony), estabilización de imagen en vídeo (Matshushita-Panasonic), enfoque automático en las cámaras de vídeo (Sanyo-Fisher, Canon), reconocimiento de la escritura (Sony Palm Top), etc.

\section{PLANTEAMIENTO DEL PROBLEMA}

Se han planteado diversos problemas a la hora de realizar un modelo basado en lógica difusa que permita obtener una predicción de la temperatura del arrabio en un horno alto.

La gran cantidad de variables de entrada por la soplante (humedad, caudal, oxígeno, etc.) como por el tragante (relación mineral-coque) como variables de salida ( $\% \mathrm{CO}, \% \mathrm{H}_{2}$, temperatura del arrabio, etc.) y la no automatización completa de la medición de la temperatura del arrabio, han obligado a formular el problema siguiendo las siguientes pautas:

- Se ha optado por elegir un valor de la temperatura de arrabio, de entre todas las mediciones realizadas por colada, y desechar el resto de las temperaturas medidas; ya que el proceso de colada no es continuo, la duración de ésta no es uniforme, y las medidas de la temperatura del arrabio no se toman a intervalos regulares de tiempo.

- Se ha elegido la última medida como valor representativo de la temperatura del arrabio por colada. Las medidas habituales de la temperatura del arrabio realizadas por colada son tres, cu- yos valores van aumentando progresivamente, es decir, la primera medida, normalmente, es menor que la última. Esto puede deberse a que mientras el horno esté cerrado, el arrabio situado cerca de la piquera, junto a las paredes, cede calor a los refractarios que forman el crisol y este arrabio es el primero en salir cuando se realiza la perforación de la piquera.

- Se han interpolado los valores seleccionados de la temperatura del arrabio, de tal forma que tengan el mismo período de muestreo que las otras variables, y a la vez, tenga un comportamiento suave, sin cambios bruscos, ya que en el proceso del horno alto, al ser un proceso con unas inercias muy grandes, los cambios de las variables de entrada afectan de una forma suave a la temperatura del arrabio. El resto de las variables (humedad, caudal del soplo, inyección de carbón, oxígeno, temperatura del soplo y relación mineral-coque) se han medido de una forma automatizada y con un período de muestreo homogéneo (10 min.).

Con la experiencia de los trabajadores de planta, se han seleccionado las variables de entrada (cuyas unidades son las típicas de un horno alto español: humedad, en $\mathrm{gr} / \mathrm{Nm}^{3}$; inyección de carbón, en $\mathrm{t} / \mathrm{h}$; caudal, en $\mathrm{Nm}^{3} / \mathrm{h}$; oxígeno, en $\mathrm{Nm}^{3} / \mathrm{h}$; temperatura del soplo, en ${ }^{\circ} \mathrm{C}$; relación mineral coque, y temperatura del arrabio, en ${ }^{\circ} \mathrm{C}$; variables que son las que se van a utilizar en el algoritmo de predicción, teniendo en cuenta la importancia que tienen éstas en el control del funcionamiento de un horno alto. Este algoritmo de predicción está compuesto a su vez por dos algoritmos: uno de entrenamiento y otro de validación. Ambos utilizan un conjunto de medidas históricas de las variables de entrada para predecir un valor futuro de la serie temporal, en este caso, de la temperatura del arrabio.

Estas variables se han incorporado al modelo con unos retrasos determinados por los trabajadores del horno según su experiencia que se producen al realizar un cambio en cualquiera de las variables de entrada al proceso citadas, ya que transcurre un determinado tiempo hasta que tiene influencia en la temperatura del arrabio. Estos retrasos están especificados en la tabla I. Hay una excepción con la temperatura del arrabio en retraso, en la que se han realizado distintas pruebas buscando una predicción de la temperatura, en la que para un retraso de 4 períodos de muestreo ( $40 \mathrm{~min}$ ) se han obtenido unos resultados bastante buenos. Estos 
Tabla I. Retrasos de las variables de entrada

Table I. Input variable delays

\begin{tabular}{ll}
\hline Variable de entrada & Retraso $(\mathbf{h})$ \\
\hline Humedad & 2 \\
Inyección de carbón & 2 \\
Caudal & 2 \\
Oxígeno & 2 \\
Temperatura del soplo & 4 \\
Relación mineral-coque & 8 \\
Temperatura del arrabio en retraso & $0,67(40 \mathrm{~min})$
\end{tabular}

resultados empeoraban visiblemente para retrasos mayores $^{[2]}$.

Además de las variables, se han añadido las necesarias para formar el vector de regresión (1), pero con un retardo de uno y dos períodos de muestreo, resultando, por tanto, tres variables de entrada por cada una de las variables elegidas anteriormente, teniendo al final un total de $21 \mathrm{va}$ riables de entrada y una salida (la temperatura del arrabio). Si se define la temperatura del arrabio en función de las entradas al modelo se tiene el siguiente sistema NARX ${ }^{[3]}$.

$$
\begin{gathered}
\hat{y}(t+1)=\mathrm{f}\left(y\left(t-\tau_{\mathrm{k}}\right), y\left(t-\tau_{\mathrm{k}}-1\right), y\left(t-\tau_{\mathrm{k}}-2\right), \ldots\right. \\
\left.\ldots, u_{\mathrm{i}}\left(t-\tau_{\mathrm{k}}\right), u_{\mathrm{i}}\left(t-\tau_{\mathrm{k}}-1\right), u_{\mathrm{i}}\left(t-\tau_{\mathrm{k}}-2\right)\right), \\
i=1, \ldots, 6 ; \quad k=1, \ldots, 7
\end{gathered}
$$

donde:

$\hat{y}(t+1)=$ valor de la predicción de la temperatura del arrabio.

$y()$ = salida del proceso (medidas de la temperatura del arrabio).

$u_{\mathrm{i}}()=$ entradas del proceso.

$\tau_{\mathrm{k}} \quad=$ retrasos de cada una de las variables, teniendo en cuenta los retrasos comentados anteriormente (tabla I).

Tanto las $y()$ como las $u_{\mathrm{i}}()$ son las entradas al modelo de lógica difusa elegido.

\section{MODELO UTILIZADO}

De entre los distintos tipos de sistemas difusos con los que se puede operar, se ha elegido el de Takagi-Sugeno-Kang ${ }^{[4]}$, ya que permite entrenar el modelo mediante dos herramientas que están integradas dentro del paquete de software de lógica difusa usado: el ANFIS y el SUBTRACTIVE
CLUSTERING (Agrupación Sustractiva). Este sistema difuso utiliza las siguientes reglas SI-ENTONCES difusas:

$$
\begin{gathered}
\text { SI } x_{1} \text { es } C_{1}^{\mathrm{k}} y \ldots y \quad x_{\mathrm{n}} \text { es } \mathrm{C}_{\mathrm{n}}^{\mathrm{k}}, \\
\text { ENTONCES } y^{\mathrm{k}}=c_{0}^{\mathrm{k}}+c_{1}^{\mathrm{k}} \cdot x_{1}+\ldots+c_{\mathrm{n}}^{\mathrm{k}} \cdot x_{\mathrm{n}}
\end{gathered}
$$

donde:

$C_{i}^{k}=$ son conjuntos difusos definidos en el sistema difuso.

$c_{\mathrm{i}}^{\mathrm{k}}=$ son constantes.

$x_{\mathrm{i}}=$ son los valores que toman las entradas del modelo (" $y()$ )" y " $u_{\mathrm{i}}($ )" del regresor).

$y^{\mathrm{k}}=$ es el valor estimado de la temperatura del arrabio para la regla $k$.

$k=1,2, \ldots, M(M=$ número de reglas $)$.

La estructura del sistema difuso se muestra en la figura 1 , a la que se ha aplicado los dos algoritmos de entrenamiento siguientes ${ }^{[5]}$ :

- Generación de una matriz de inferencia difusa general y aplicación posterior del ANFIS (Sistema de Inferencia Neuro-Difuso Adaptativo) $)^{[6,7]}$.

- Aplicación del agrupamiento sustractivo ${ }^{[7,8]}$.

El primer algoritmo se ralentiza cuando el proceso se vuelve más complejo, ya que el cálculo aumenta exponencialmente con la dimensión del problema. En él, la generación de la matriz del sistema de inferencia difuso se realiza sin ningún tipo de entrenamiento, por tanto, tiene una estructura general construyendo reglas difusas de la forma de (2) para cada una de las posibles combinaciones con todas las variables de entrada y con todas las funciones de pertenencia ${ }^{[9]}$ asociadas a cada una de las variables de entrada. Este hecho provoca

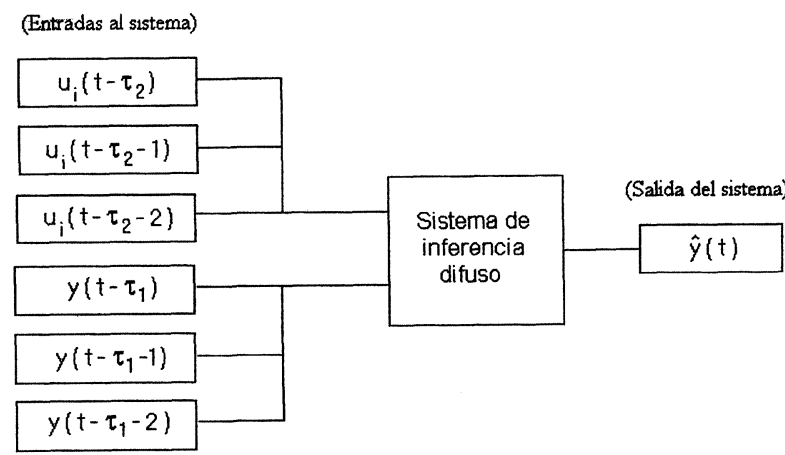

Figura 1. Esquema del sistema difuso.

Figure 1. Fuzzy system scheme. 
que el número de reglas sea muy elevado cuando el número de entradas es muy grande, haciendo imposible un manejo sencillo y rápido de esta matriz durante el período de entrenamiento (aplicación del Sistema de Inferencia Neuro-Difuso Adaptativo). Al final del entrenamiento se obtienen $\operatorname{los} c_{\mathrm{i}}^{\mathrm{k}}$ de las reglas (2).

El segundo algoritmo es más rápido que el primero ya que el cálculo sólo es proporcional al número de datos e independiente de la dimensión del problema que se está considerando. En él, la generación de la matriz del sistema de inferencia difuso se realiza mediante un entrenamiento inicial, anterior a su construcción, buscando relaciones entre las entradas y las salidas a priori y formando agrupaciones entre los datos que tienen un comportamiento similar, reduciendo, por tanto, el número de reglas difusas (2) al número de agrupaciones encontradas. Así, en la matriz de inferencia difusa, cada una de las variables de entrada tendrá tantas funciones de pertenencia como agrupaciones se hayan encontrado y, por tanto, también se creará el mismo número de reglas difusas. Un factor muy importante es el radio de influencia, que es un parámetro del algoritmo de entrenamiento, cuyo valor se determina mediante prueba y error: cuanto menor es el radio de influencia, se obtiene un mayor número de agrupaciones y, por ello, se crea un mayor número de reglas y de funciones de pertenencia asociadas a cada una de las variables de entrada, consiguiendo un sistema más complejo y más preciso. Al final del entrenamiento se obtiene un número de reglas y los $c_{\mathrm{i}}^{\mathrm{k}}$ de éstas (2).

El entrenamiento se ha realizado con 12.000 datos, mientras que la validación se ha realizado con 5.000. A pesar de que los resultados obtenidos con el primer algoritmo han sido aceptables, en este trabajo se ha utilizado el segundo, ya que estos resultados han sido mejores, como se observa en la figura 2 para el entrenamiento, y en la figura 3 para la validación. En las figuras 2a y 3 a se observa que las predicciones no están retrasadas con respecto a la medida real de la temperatura del arrabio, donde los círculos representan los valores medidos de la temperatura del arrabio y la línea continua representa la predicción de ésta. Las figuras $2 b$ y $3 b$ muestran el error entre la medida real y la predicción de la temperatura del arrabio del entrenamiento y de la validación, respectivamente. Se observa que este error es menor de $10 \mathrm{~K}$, que es el margen de error que tiene el termopar con el que se ha realizado la medida de la temperatura del arrabio. En la figura 3a se observa que la predicción no está retrasada con respecto a la medida real. En ambas figuras, la unidad de muestreo es de $10 \mathrm{~min}$.
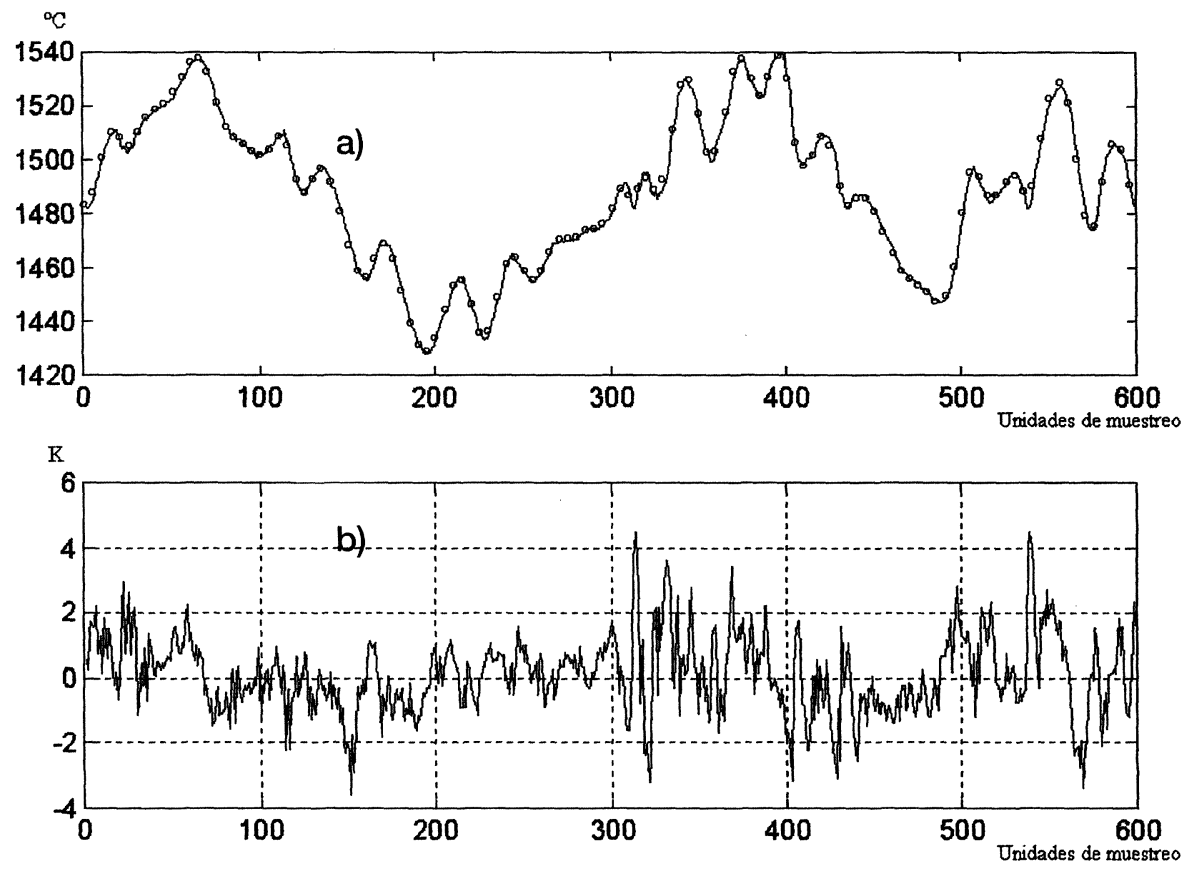

Figura 2. Predicción del sistema difuso con el conjunto de entrenamiento. a) Entrenamiento de la temperatura del arrabio. b) Error de predicción con el conjunto de entrenamiento.

Figure 2. Fuzzy system prediction with training set. a) Hot metal training. b) Prediction error with training set. 

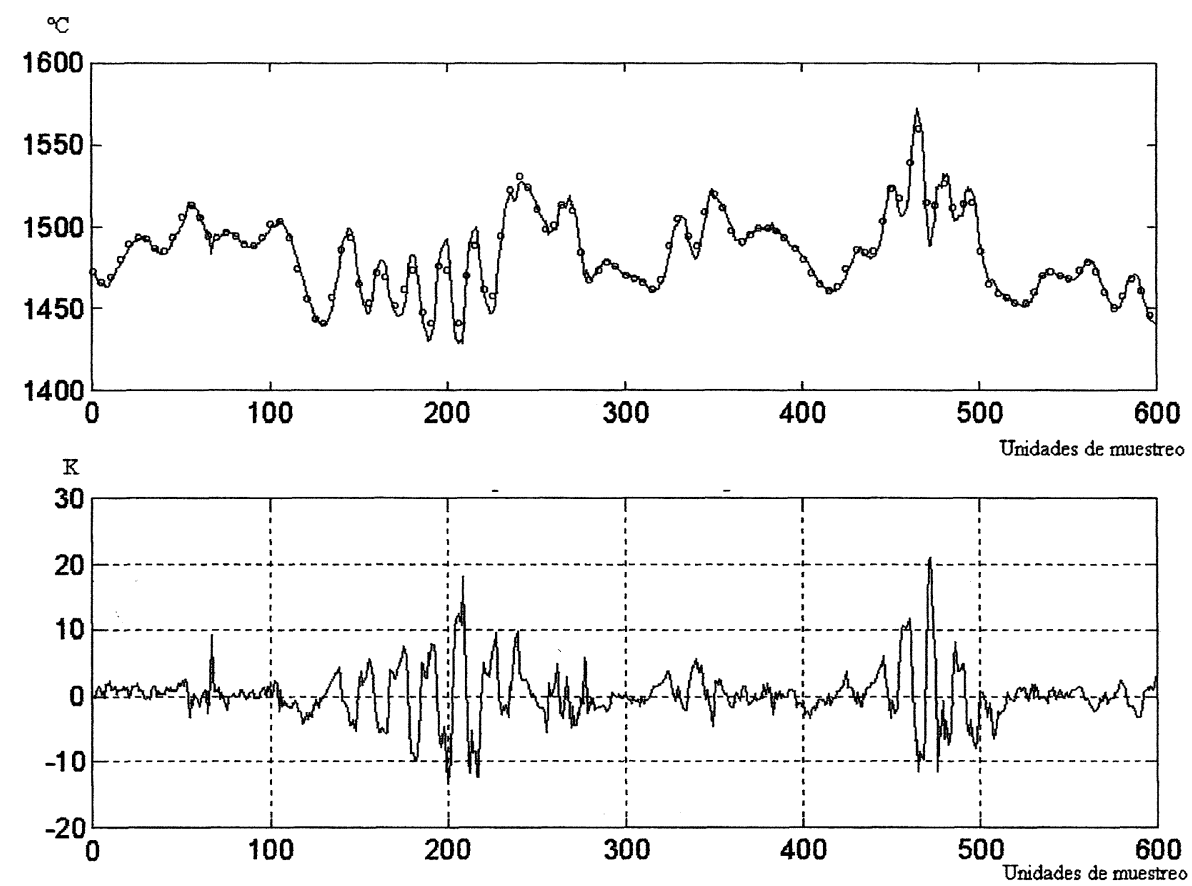

Figura 3. Predicción del sistema difuso con el conjunto de validación. a) Validación de la temperatura del arrabio. b) Error de predicción con el conjunto de validación.

Figure 3. Fuzzy system prediction with validation set. a) Hot metal validation. b) Prediction error with validation set.

\subsection{Simulación}

En la figura 4 se muestra el esquema del simulador desarrollado basado en el regresor anteriormente descrito (1). Las entradas al sistema van seguidas de un bloque que es el encargado de retrasar estas entradas los tiempos que se indican en la tabla I. La temperatura del arrabio en retraso, que es una realimentación en lazo cerrado de las predicciones del sistema, va seguido de su correspondiente

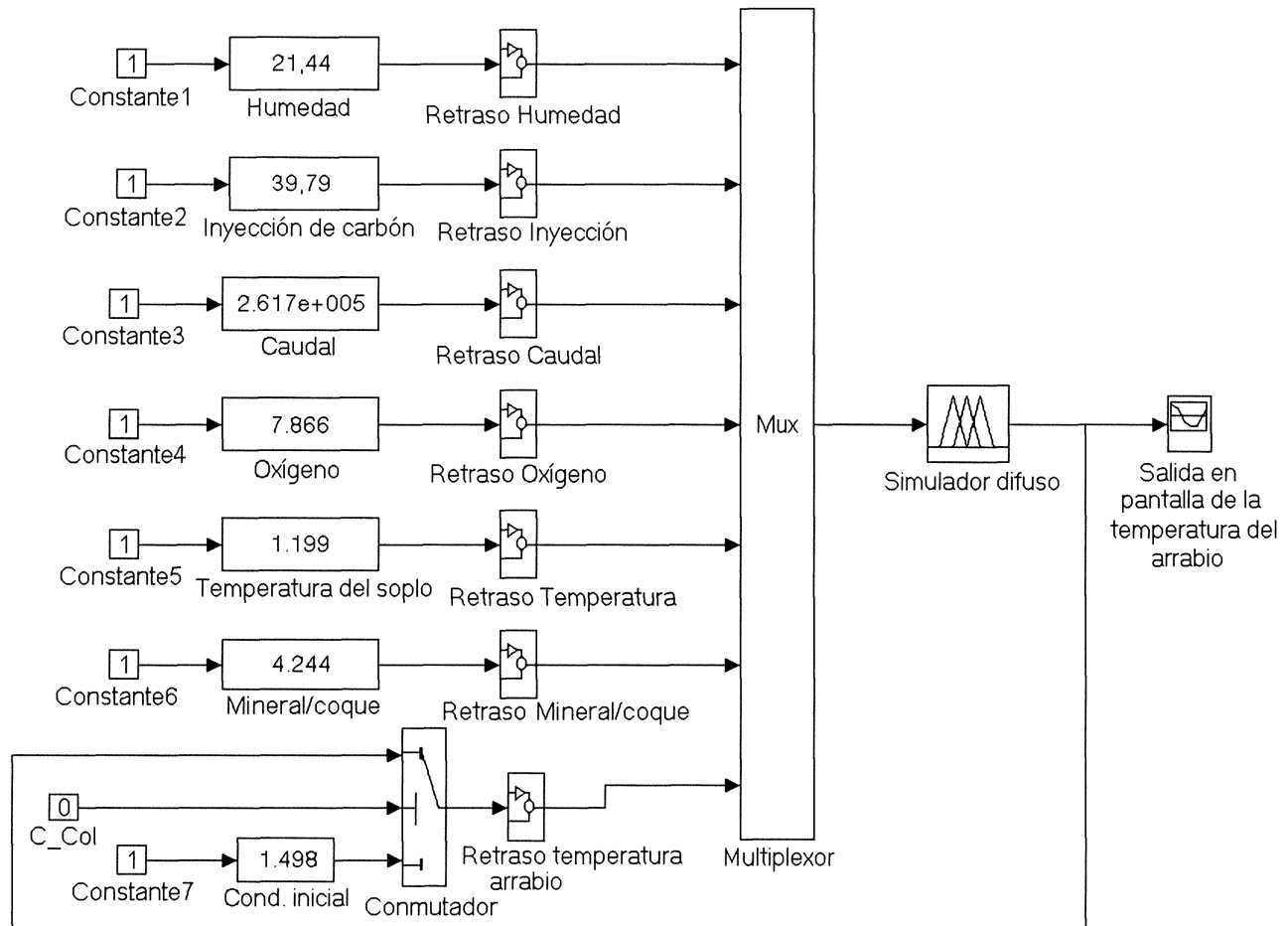

Figura 4. Esquema del simulador difuso.

Figure 4. Fuzzy simulator scheme.

44

(c) Consejo Superior de Investigaciones Científicas

Licencia Creative Commons 3.0 España (by-nc) 
retraso (4 unidades de muestreo como se dijo anteriormente). Todas las entradas confluyen en el sistema de inferencia difusa (bloque simulador difuso) que es el que proporcionará la predicción de la temperatura del arrabio.

\section{RESULTADOS}

Una vez obtenido el esquema del simulador del horno alto, se han realizado distintos ensayos en los que se han mantenido constante todas las variables de entrada menos una, cuyo valor se ha ido aumentando y disminuyendo para realizar un análisis de sensibilidad del comportamiento de la temperatura del arrabio con respecto a la variable que se está modificando. Este ensayo se ha repetido para cada una de las entradas al simulador.

La figura 5 a muestra las acciones que se han realizado sobre la humedad en distintos ensayos en los que se ha aumentado y disminuido un 10 , un 15 y un $20 \%$ el valor de dicha variable. La figura $5 \mathrm{~b}$ representa el comportamiento que ha tenido la temperatura del arrabio al realizar esas acciones sobre la humedad. Se observa que, al aumentar la humedad, la temperatura del arrabio disminuye y viceversa. La figura 6a representa, al igual que la figura 5 a con la humedad, los distintos ensayos realizados en los que se ha aumentado y disminuido $2 \mathrm{t} / \mathrm{h}$ y 4 t/h la inyección de carbón. La figura $6 \mathrm{~b}$ muestra el comportamiento de la temperatura del arrabio a los cambios realizados en esta variable en donde, al aumentar la inyección de carbón, aumenta la tem-
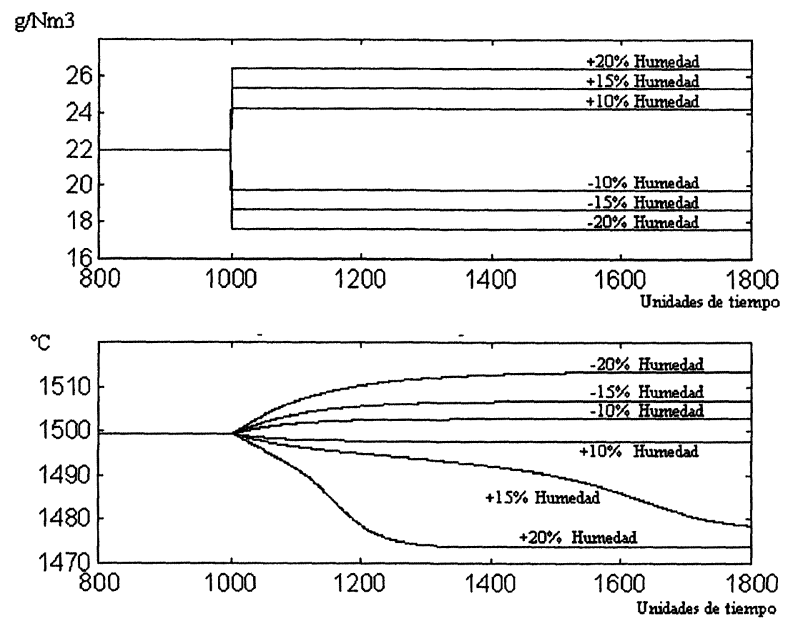

Figura 5. Respuesta de la temperatura del arrabio a los cambios en la humedad. a) Variación de la humedad. b) Comportamiento de la temperatura del arrabio.

Figure 5. Hot metal temperature response to moisture changes. a) Blast moisture variation. b) Hot metal behaviour.
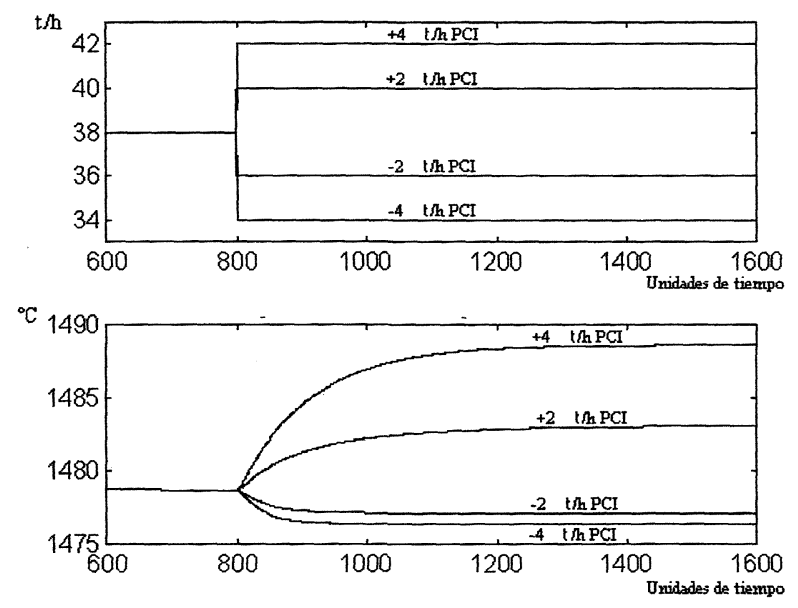

Figura 6. Respuesta de la temperatura del arrabio a los cambios en la inyección de carbón. a) Variación de la inyección de carbón. b) Comportamiento de la temperatura del arrabio.

Figure 6. Hot metal temperature response to $\mathrm{PCl}$ changes. a) Coal inyection variation. b) Hot metal behaviour.

peratura del arrabio y viceversa. Estos ensayos también se han repetido para el caudal, cuyos resul- tados se han reflejado en las figuras $7 \mathrm{a}$ y $7 \mathrm{~b}$.

En las figuras 5, 6 y 7, se puede observar que el comportamiento de la temperatura del arrabio para cada una de las variables modificadas es el que se esperaba, ya que estos comportamientos han sido facilitados por los que trabajan en el horno alto. Se observa también unas grandes inercias en la temperatura del arrabio al tardar bastantes unidades de tiempo en estabilizarse, teniendo en cuenta que una unidad de muestreo es equivalente a 10 min. En estos resultados se puede observar que la
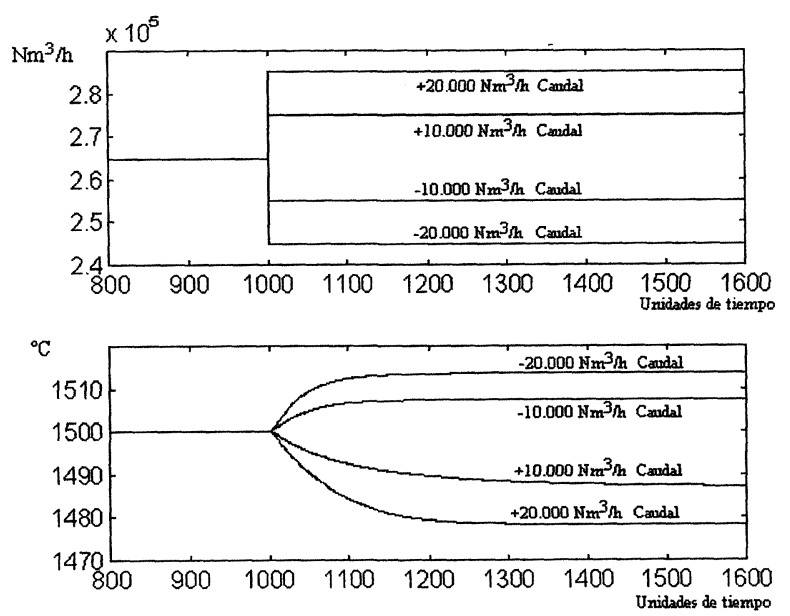

Figura 7. Respuesta de la temperatura del arrabio a los cambios en el caudal del soplo. a) Variación del caudal del soplo. b) Comportamiento de la temperatura del arrabio.

Figure 7. Hot metal temperature response to blast volume changes. a) Blast volume variation. b) Hot metal behaviour. 
reacción de la temperatura del arrabio es inmediata a la acción realizada sobre la variable en cuestión; esto se debe a que los retrasos anteriormente mencionados (Tabla I) ya se han tenido en cuenta en la estructura del simulador.

Con el resto de las variables estudiadas (oxígeno, temperatura del soplo y relación mineral-coque), el comportamiento de la temperatura del arrabio también ha sido el supuesto.

\section{CONCLUSIONES}

El modelo desarrollado es capaz de estimar razonablemente la temperatura del arrabio y el comportamiento de ésta ante cualquier modificación de las variables de entrada al modelo y dentro del rango operativo de cada una de ellas.

El poder predecir la temperatura de arrabio con 40 min. de antelación, sirve como ayuda a los que trabajan en el horno alto para evitar los sobrecalentamientos y los enfriamientos que puede sufrir éste, es decir, sirve como un sistema de alerta para el horno alto.

\section{Agradecimientos}

Los autores agradecen a la Comunidad Europea del Carbón y el Acero y a la CICYT la financiación económica del proyecto Investigation of Fuzzy
Logic and Neural Network Based Strategies for Control Optimisation of Iron Process dentro del cual se enmarca el presente trabajo, así como a la empresa Aceralia y, más concretamente, a los Drs. B. Fernández y J. Sainz por su colaboración.

\section{REFERENCIAS}

[1] J.M. Mendel, Proc. IEEE, vol. 83, 3, 1995, pp. 345377 ,

[2] J. JiMÉNEZ, J. MOCHÓN Y A. FORMOSO, Investigation of Fuzzy Logic and Neural Network Based Strategies for Control Optimisation of Iron Process, ECSC Agreement 7210-AA/ 940, Draft Final Report, 1999.

[3] M.A. Henson y D.E. Seborg, Nonlinear Process Control, Prentice-Hall Inc., New Jersey EE.UU., 1997.

[4] LI-XIN WANG, Adaptive Fuzzy Systems and Control: Design and Stability Analysis, Prentice-Hall International, New Jersey, EE.UU., 1994, pp. 29-48,

[5] N. GULLEY Y JYH SHING R. JANG, Fuzzy Logic Toolbox for use with Matlab. User's Guide, The Math Works Inc., '1995, pp 3-57 a 3-59.

[6] JYH-SHING R. JANG, IEEE Trans. Neural Networks, 3 (1992.) 714-723.

[7] JyH-Shing R. JANG, C.-T. SUn y E. Mizutani, NeuroFuzzy and Soft Computing, Prentice-Hall Inc., New Jersey, EE.UU., 1997.

[8] R. YAGER y D. FILEV, J. Intelligent Fuzzy Systems, 2 (1994) 209-219.

[9] L-XIN WANG, A Course in Fuzzy Systems and Control, Prentice-Hall International, Inc., New Jersey, EE.UU. 1997. 